\title{
Cognitive Dynamic Optical Networks
}

de Miguel, Ignacio; Duran, Ramon J.; Lorenzo, Ruben M.; Caballero Jambrina, Antonio; Tafur Monroy, Idelfonso ; Ye, Yabin; Tymecki, Andrzej; Tomkos, Ioannis; Angelou, Marianna; KIonidis, Dimitrios Total number of authors:

13

Published in:

2013 Optical Fiber Communication Conference and Exposition and the National Fiber Optic Engineers Conference (OFC/NFOEC)

Link to article, DOI:

10.1364/OFC.2013.OW1H.1

Publication date:

2013

Document Version

Publisher's PDF, also known as Version of record

Link back to DTU Orbit

Citation (APA):

de Miguel, I., Duran, R. J., Lorenzo, R. M., Caballero Jambrina, A., Tafur Monroy, I., Ye, Y., Tymecki, A., Tomkos, I., Angelou, M., Klonidis, D., Francescon, A., Siracusa, D., \& Salvadori, E. (2013). Cognitive Dynamic Optical Networks. In 2013 Optical Fiber Communication Conference and Exposition and the National Fiber Optic Engineers Conference (OFC/NFOEC) [OW1H.1] IEEE. https://doi.org/10.1364/OFC.2013.OW1H.1

\section{General rights}

Copyright and moral rights for the publications made accessible in the public portal are retained by the authors and/or other copyright owners and it is a condition of accessing publications that users recognise and abide by the legal requirements associated with these rights.

- Users may download and print one copy of any publication from the public portal for the purpose of private study or research.

- You may not further distribute the material or use it for any profit-making activity or commercial gain

- You may freely distribute the URL identifying the publication in the public portal 


\title{
Cognitive Dynamic Optical Networks
}

\author{
Ignacio de Miguel ${ }^{1}$, Ramón J. Durán ${ }^{1}$, Rubén M. Lorenzo ${ }^{1}$, Antonio Caballero², Idelfonso Tafur Monroy ${ }^{2}$, \\ Yabin Ye ${ }^{3}$, Andrzej Tymecki ${ }^{4}$, Ioannis Tomkos ${ }^{5}$, Marianna Angelou ${ }^{5}$, Dimitrios Klonidis ${ }^{5}$, \\ Antonio Francescon ${ }^{6}$, Domenico Siracusa $^{6}$, Elio Salvadori ${ }^{6}$ \\ 1: University of Valladolid - UVa (Spain), 2: Technical University of Denmark - DTU (Denmark), 3: Huawei Technologies (Germany), \\ 4: Orange Labs Poland (Poland), 5: Athens Information Technology center-AIT (Greece), 6: CREATE-NET (Italy) \\ ignacio.miguel@tel.uva.es
}

\begin{abstract}
Cognitive networks are a promising solution for the control of heterogeneous optical networks. We review their fundamentals as well as a number of applications developed in the framework of the EU FP7 CHRON project.

OCIS codes: (060.4250) Networks; (060.4510) Optical communications
\end{abstract}

\section{Introduction}

Optical networks are facing increased levels of heterogeneity, from types of services to transmission technologies. Hence, a key issue of highly heterogeneous networks is how to efficiently control and manage network resources while fulfilling user demands and complying with quality of service requirements. A solution for such a scenario may come from cognitive networks. A cognitive network is defined as "A network with a process that can perceive current network conditions, and then plan, decide, and act on those conditions. The network can learn from these adaptations and use them to make future decisions, all while taking into account end-to-end goals." [1]. Hence, there are three main ingredients in such a network:

- Monitoring elements, which provide the network with the perception of the current conditions, and thus enable an aware network.

- Software adaptable elements, which provide the network with the capacity of modifying its current configuration, thus enabling an adaptive network.

- Cognitive processes, which learn or make use of past history, so that even when facing two equivalent scenarios, the network (or the element containing those cognitive processes) may act in a different way if its previous history is different. This third element is the main feature which enables a cognitive network.

Therefore, a cognitive network is a network which is able to adapt itself to current or forecasted conditions by taking into account previous history, and which is able to act proactively, rather than reactively, in order to avoid problems before they arise. Moreover, those tasks should be performed autonomously, with little or no intervention of the network operator. Cognitive networks have already shown their advantages in wireless environments [2], but they are also applicable to wired communication architectures, and are especially appealing for optimizing performance in heterogeneous networks. Thus, in this paper we describe how cognition can be applied in the framework of optical networking.

\section{Architectures for cognitive optical networks}

The design of a cognitive optical network involves determining how the three aforementioned ingredients are implemented (and where), how they are glued together, as well as determining which tasks are going to be solved with the help of cognition. An initial answer to some of these issues is provided by a set of cognitive architectures or frameworks proposed in the literature, such as the work by Thomas et al. [1] and Kliazovich et al. [3], targeted to generic cognitive networks, or the proposals by Zervas and Simeonidou [4], Wei et al. [5], and the CHRON project approach (Cognitive Heterogeneous Reconfigurable Optical Network) [6,7], targeted at cognitive optical networks.

These architectures show that cognition can be implemented in different dimensions, in terms of devices and protocol layers. For instance, in a cognitive network implementation, software-defined transceivers may include monitoring functionalities together with internal intelligence to modify their configuration autonomously, i.e., being truly cognitive transceivers. However, another implementation may opt for shifting the intelligence in charge of configuring those transceivers to the upper layers of the nodes where the transceivers are located, thus being the network nodes the cognitive elements rather than the transceivers themselves. That example may find its way in a network with distributed cognition, where all network nodes are equipped with cognitive capabilities and collaborate in sharing acquired knowledge. Nevertheless, another possibility is a network with centralized cognition, where a single node (the control node) contains the intelligence and makes decisions which are then communicated to the remaining network nodes by means of control and management plane protocols with suitable extensions. 
On the other hand, the level and type of cognition to be added to a network is dependent, not only in the adopted approach, as we have just described, but also on the capabilities of network monitors and software-adaptable elements employed: the higher the flexibility of the available software-adaptable elements, the higher the potential of cognition. However, although the utilization of software-defined networks, software-defined transceivers and flexible (or elastic) networks is usually associated with cognitive optical networks, it should be noted that these technologies are not strictly necessary for adopting a cognitive networking approach.

As an example, the CHRON project [6,7] has proposed a distributed and a centralized architecture for cognitive optical networks but has mainly focused on the latter one, shown in Fig. 1(a). The core element of the CHRON cognitive architecture is the cognitive decision system (CDS). The CDS receives traffic demands, and determines how to handle them by taking into account both the current status of the network and past history, and instructs the control plane to configure network elements accordingly. Therefore, the CDS is complemented with a network monitoring system, which provides traffic status and optical quality of transmission measurements, and with a set of GMPLS-based control and management mechanisms to implement the decisions that are made by the CDS and to disseminate the monitored information. The CDS is involved in very diverse tasks related to network control and optimization. Hence, rather than implementing the whole CDS as a monolithic module, it is divided into different modules, each offering a functionality (or a set of related functionalities), and all of them exploiting cognition, as shown in Fig. 1(a) [6].

\section{Applications of cognition in optical networks}

Cognition may find many diverse applications in optical networks. In this section, we review three applications of cognition proposed within the CHRON context.

One of the modules of the CDS is the quality of transmission (QoT) estimator. This module makes a prediction of signal quality of new lightpaths to be established in the network (as well as of the impact on existing connections) by using past history. Moreover, the module receives feedback from the network monitoring system, which may be used to update its knowledge base and thus adapt to changing conditions like component ageing. The cognitive operation of this module relies on the utilization of data mining techniques. For instance, Fig. 1(b) shows the percentage of successful classifications of lightpaths into high or low QoT categories when using different techniques such as a naive Bayes classifier, different types of decision trees, and a case-based reasoning (CBR) approach for the 34-node GÉANT2 dispersion-compensated network, equipped with 64 wavelengths. As shown in the figure, and as demonstrated in [8], the CBR approach achieves more than $99 \%$ successful classifications of optical connections, and is much faster for on-line operation than an existing non-cognitive approach, thus demonstrating the advantages of cognition.
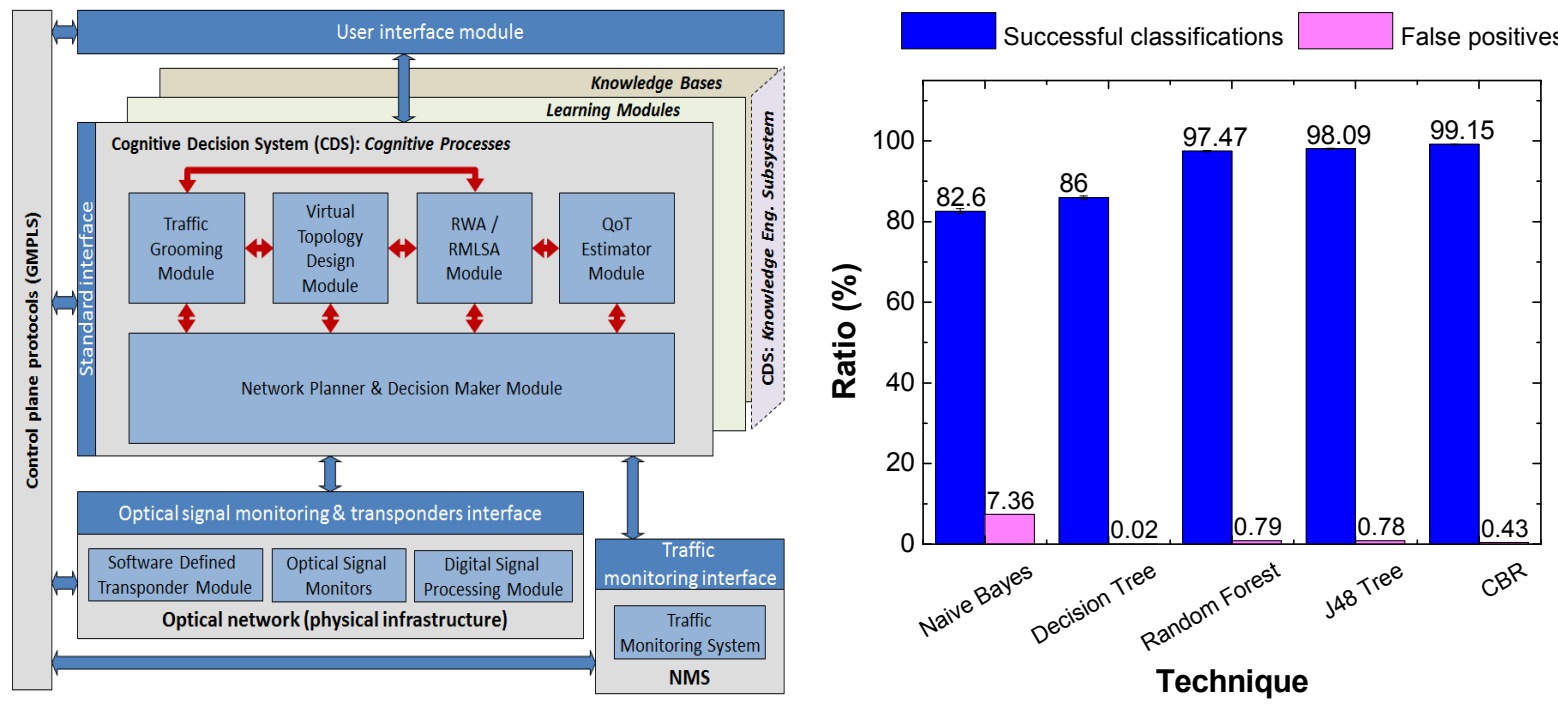

Fig. 1. (a) The CHRON schematic architecture (for a network with centralized cognition). (b) Percentage of successful classification and false positives obtained by different data mining techniques when used for QoT assessment in the GÉANT2 network.

A second example of the potential of cognition in optical networks is related to the virtual topology design module. In [9] we have proposed a multiobjective genetic algorithm to design impairment-aware and survivable virtual topologies with the aim of reducing both the energy consumption and the network congestion. In a single 
execution, the algorithm provides several solutions with different trade-offs in terms of the two optimization objectives just mentioned. That method has been further enhanced with two cognitive techniques based on the utilization of memory to remember solutions successfully used in the past, and a Tabu list to remember connections with low QoT. We have studied the performance of this method in the transparent Deutsche Telekom network, and assumed a traffic-varying environment where the virtual topology is reconfigured every hour. By means of simulation, we have demonstrated that the inclusion of cognition leads to finding more and better solutions, as shown in Fig. 2(a), and moreover, that the network learns as time passes, since results improve with time.

The third and last example is a step forward towards more autonomous networks, where cognition resides on transceivers rather than on a control node. Thus, in [10] we have proposed a cognitive digital receiver which, by means of clustering algorithms, is able to identify the incoming signal format (QPSK/8PSK/16QAM) without the need of receiving a prior control message, thus opening the door to the autonomous modification of the modulation format, as shown in Fig. 2(b).
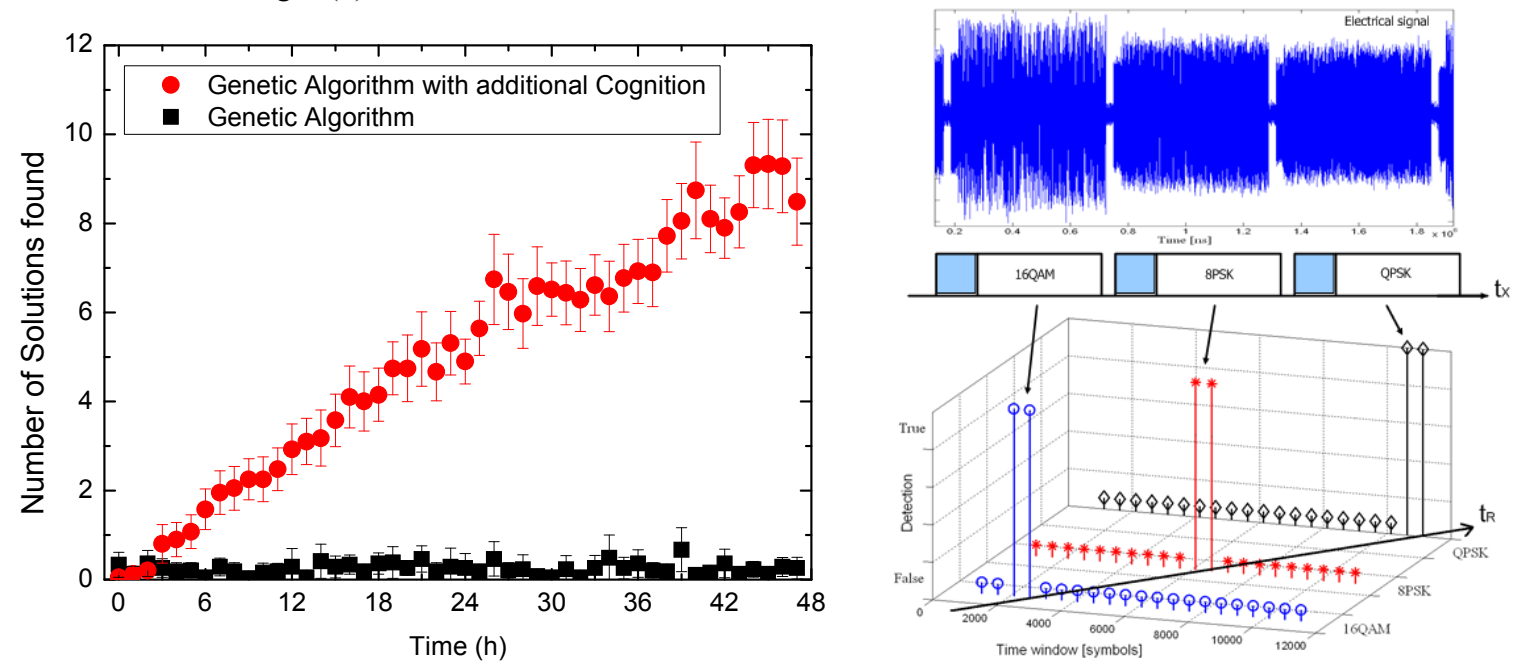

Fig. 2. (a) Number of solutions found depending on the time in which the request to design the virtual topology is created. (b) Cognitive signal modulation format recognition for three data payloads with alternated QPSK, 8PSK and 16QAM modulation formats

\section{Summary}

There are many different alternatives for the implementation of cognition in optical networks. In this paper, besides discussing the fundamentals of cognition, we have briefly described the approach followed by the EU FP7 CHRON project, whose aim is to efficiently control heterogeneous optical networks (in terms of services and physical layer interfaces) by exploiting cognitive techniques, and have shown three cognitive applications developed in the project.

\section{Acknowledgments}

The CHRON project (Cognitive Heterogeneous Reconfigurable Optical Network) has received funding from the European Community's Seventh Framework Programme [FP7/2007-2013], under grant agreement $n^{\circ} 258644$.

\section{References}

[1] R.W. Thomas et al., "Cognitive networks: Adaptation and learning to achieve end-to-end performance objectives," IEEE Communications Magazine, pp. 51-57, Dec. 2006.

[2] Q.H. Mahmoud (ed.), Cognitive Networks: Towards Self-Aware Networks (John Wiley \& Sons, Ltd., 2007).

[3] D. Kliazovich, F. Granelli, and N. L. S. Da Fonseca, "Architectures and cross-layer design for cognitive networks" in Handbook of sensor networks (World Scientific Publishing Co, 2010), Chap. 1.

[4] G. S. Zervas and D. Simeonidou, "Cognitive optical networks: Need, requirements and architecture," in Proc. ICTON 2010, paper We.C1.3.

[5] W. Wei, C. Wang, and J. Yu, "Cognitive optical networks: key drivers, enabling techniques, and adaptive bandwidth services," IEEE Communications Magazine, pp. 106-113, Jan. 2012.

[6] EU FP7 CHRON project, http://www.ict-chron.eu

[7] I. Tomkos et al., "Next generation flexible and cognitive heterogeneous optical networks," in The Future Internet - Future Internet Assembly

2012: From Promises to Reality (Springer, 2012), pp. 225-236.

[8] T. Jiménez et al., "A cognitive system for fast quality of transmission estimation in core optical networks," Proc. OFC 2012, paper OW3A.5.

[9] N. Fernández et al., "Survivable and impairment-aware virtual topologies for reconfigurable optical networks: a cognitive approach," in Proc. RNDM 2012, pp. 183-189.

[10] N. Guerrero Gonzalez, D. Zibar, and I. Tafur Monroy, "Cognitive digital receiver for burst mode phase modulated radio over fiber links," in Proc. ECOC 2010, paper P6.11. 